\title{
On-Site Predominant Method of Observer Status in the Echo-Signals Parametric Space
}

Igor V. Lyutikova, Vladimir A. Kopylova and Valeriy V. Zamaraev ${ }^{a}$ Siberian Federal University 79 Svobodny, Krasnoyarsk, 660041, Russia ${ }^{b} A S D$ troops Central Research Institute of the Russian Federation Defense Ministry 32 Nabereznaya Afanasiya Nikitina, Tver, 170026, Russia

Received 03.03.2016, received in revised form 17.04.2016, accepted 06.05.2016

The article suggests the total method based on current information on the maintenance mode and signs of maneuvering targets, allowing to assess the situation, open training and the willingness of the enemy to launch interceptor missiles into far dogfight. Shows the relevance of the further implementation of the method is based upon the development of additional algorithms of decision-making support pilot on the defensive and offensive actions (selecting RADAR modes) in information-management systems for retrofit and advanced fighter jets to engage in preemptive provisions of observer in parametric space taken by ECHO-signals and subsequent effective attack.

Keywords: escorts, maneuvering, dogfight, attack, decision support, information management, RADAR.

Citation: Lyutikov I.V., Kopylov V.A., Zamaraev V.V. On-site predominant method of observer status in the echo-signals parametric space, J. Sib. Fed. Univ. Eng. technol., 2016, 9(4), 481-488. DOI: 10.17516/1999-494X-2016-9-4-481-488.

(C) Siberian Federal University. All rights reserved

* Corresponding author E-mail address: lyutikovigor@mail.ru 


\title{
Метод занятия преимущественного \\ положения наблюдателя \\ в параметрическом пространстве \\ принимаемых эхо-сигналов
}

\author{
И.В. Лютиков ${ }^{a}$, В.А. Копылов ${ }^{a}$, В.В. Замараев ${ }^{0}$ \\ ${ }^{a}$ Сибирский федеральный университет \\ Россия, 660041, Красноярск, пр. Свободный, 79

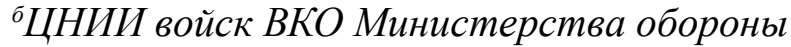 \\ Российской Федераиии \\ Россия, 170026, Тверь, ул. Набережная Афанасия Никитина, 32
}

\begin{abstract}
В статье предложен общчий метод на основе текущей информации режима сопровождения и признаков маневрирования ичелей, позволяющий оценить обстановку, вскрыть подготовку u готовность перехватчиков противника к пуску ракет в дальнем воздушном бою. Показана актуальность дальнейшей реализации дополнительных алгоритмов поддержки принятия решений летчика на оборонительные и наступательные действия (выбор режимов работы БРЛС) в информацчионно-управляюших системах модернизируемых и перспективных истребителей для занятия преимуществвенного положения наблюдателя 6 параметрическом пространстве принимаемых эхо-сигналов и последующей эффективной атаки.
\end{abstract}

Ключевые слова: сопровождение, маневрирование, воздушный бой, атака, поддержка принятия решений, информационно-управляющяая, БРЛС.

Известно, что как в ходе подготовки, так и в ходе ведения воздушного боя лётчик анализирует воздушную обстановку, которая включает в себя в том числе оценку степени опасности целей, вскрытие их действий по подготовке к пуску ракет и т.д. [1, 2]. Лётчик должен оценить, имеет ли он преимущество перед противником по пуску ракеты в текущих условиях, т. е. взаимное расположение своего самолёта и самолёта противника, скорости обоих самолётов, тип готовящихся к применению ракет.

Если истребитель не имеет преимущества, лётчик определяет условия, в которые должен быть выведен истребитель для обеспечения преимущества, выбирает оптимальный способ маневрирования для достижения этих условий, оценивает, успеет ли самолёт их достигнуть при выбранном способе маневрирования до того, как противник войдёт в свою зону возможных пусков (ЗВП) и сможет пустить ракету.

Естественно желание помочь лётчику в решении этой задачи путём создания соответствующего алгоритмического обеспечения. Для решения этой задачи в первую очередь необходимо привести понятия, которые описывают бой (или атаку).

Атака - это совокупность взаимосвязанных в пространстве и времени действий группы перехватчиков, направленных на достижение определённой цели (обычно это уничтожение противостоящего противника). Поэтому целесообразно атаку представить в виде графа, включающего в себя совокупность простых действий. Граф, таким образом, будет определять структуру атаки. Под простым действием будем понимать действие, которое может быть пред- 
ставлено известными функциями с соответствующим набором параметров. Например, прямолинейный полёт перехватчика на заданном временном интервале (линейная функция), манёвр курсом с заданными параметрами (степенная функция), постановка активных помех с заданными параметрами (ступенчатая функция) и т.д. Действия пары истребителей являются составным действием и будут отражаться в графе совокупностью векторов, каждый из которых характеризует действия отдельного перехватчика. Задача алгоритма - преобразовать текущую информацию о положении средств нападения противника и признаках их действий в оценку опасности воздушной обстановки в интересах организации боя. В свою очередь, опасность обстановки будем раскрывать с помощью прогноза возможных действий противника, параметров, описывающих эти действия, и степенью доверия к этим оценкам (достоверностью решений и оценок).

В атаке присутствуют следующие простые действия:

- действия планера;

- равномерный прямолинейный полёт $r(t)=r_{0}+v\left(t-t_{0}\right)$ (три векторных параметра описывают это действие $\left.-r_{0}, v, t_{0}\right)$;

- манёвр $r(t)=r_{0}+v\left(t-t_{0}\right)+a\left(t-t_{0}\right)^{2} / 2$ (четыре параметра $\left.-r_{0}, v, t_{0}, a\right)$ в интересах занятия выгодного положения для атаки;

- манёвр $r(t)=r_{0}+v\left(t-t_{0}\right)+a_{n p}\left(t-t_{0}\right)^{2} / 2$ (четыре параметра) в интересах противодействия атаке противника (параметры манёвра $a_{n p}$ отличаются от параметров для проведения атаки);

- действия БРЛС перехватчика:

- режим поиска целей,

- сопровождение целей,

- подсвет целей в интересах наведения ракет.

Каждый вид режима характеризуется своей совокупностью параметров (в первую очередь временных - график работы в различных режимах):

- действия передатчика постановщиков помех из состава атакующей группы;

- постановка заградительных шумовых помех;

- постановка ответно-импульсных помех;

- постановка ответных шумовых помех.

Каждый вид помех характеризуется соответствующей совокупностью параметров (временной график и параметры помехи).

При необходимости перечень простых действий может быть расширен.

Составным действием будет, например, выход в зону пуска ракет при соблюдении определённых условий, необходимых для пуска ракет (взаимосвязь с простыми действиями отражается на графе). Достижение этого состояния противником будем считать его готовностью к проведению атаки (пуску ракет).

Рассмотрение процесса вскрытия подготовки противника к атаке начнём с момента взятия цели на сопровождение. На этот момент будем считать известными положение цели в пространстве и её вектор скорости (на уровне измерений) - вектор состояния цели на $k$-й момент времени. Кроме этого, может быть получен признак одиночная цель или групповая, а также информация о помеховой обстановке в зоне действия БРЛС. 
Совокупность действий планера, соответствующая подготовке его к атаке, должна приводить планер из исходной точки на трассе полёта перехватчика (в данном случае из точки на момент захвата цели) в точку возможных пусков ракет (в ЗВП). Поэтому траекторная информация в соответствии с принятым законом движения должна анализироваться на возможность попадания текущей точки на трассе (или экстраполированной) в зону возможных пусков ракет. Функция правдоподобия по заданной выборке характеризует правдоподобность действия данного перехватчика в интересах атаки для различных тактических приёмов. Функция правдоподобия для тактического приёма рассчитывается как произведение функций правдоподобия простых событий, образующих тактический приём.

Каждый тактический приём характеризуется взаимосвязанными во времени действиями перехватчиков, входящих в его состав. Поэтому граф строится для каждого тактического приёма и соответственно рассчитывается функция правдоподобия для каждого приёма. В последующем алгоритм должен выбрать тактический приём, имеющий наибольшее значение функции правдоподобия.

Таким образом, можем получить значения функции правдоподобия как для различных оборонительных, так и наступательных тактических приёмов (свернуть всю текущую информацию с достаточно большого временного интервала к виду, необходимому для ведения боевых действий). Блок-схема алгоритма приведена на рис. 1.

Рассмотрим работу блоков модели.

Блок 1. Исходной информацией для первого блока является взаимное положение истребителя и цели (возможно, и групповой) и вектора скоростей движения цели и перехватчика. Рассмотрим в качестве примера один из возможных вариантов построения модели движения цели. Не теряя общности, рассмотрим такие условия, когда атака может производиться под любым углом пересечения траекторий перехватчика и цели. Пусть при оценке обстановки используется только координатная информация.

Пусть и цель, и перехватчик движутся прямолинейно с постоянной скоростью.

1. Рассчитаем координаты точки пересечения этих траекторий $\left(x_{0}, y_{0}\right)$.

2. Определим время полета истребителя и ракеты до этой точки, считая, что ракета пущена с максимальной дальности $T_{\text {ио. }}$

3. Определим время полёта цели до этой точки $T_{\text {ц0} \text {. }}$.

4. Если истребитель раньше проходит эту точку $\left(T_{\text {н0 }} \leq T_{\text {ц0}}\right)$, можно увеличить длину пути, совершив два манёвра в противоположные стороны и обеспечив тем самым равенство моментов времёни прихода ракеты и цели в точку $\left(x_{0}, y_{0}\right)$. Длины пути, проходимого самолётом и ракетой после её запуска, определяются из уравнения $\frac{l_{c}}{v_{c}}+t_{p}=\frac{l_{u}}{v_{u}}$. В свою очередь, путь, проходимый самолётом, включает в себя два отрезка поворота траектории с углом поворота $\alpha$ и участок движения по прямой $l_{c}=4 R \pi \alpha+l_{n p}$.

Таким образом, существует множество решений этой задачи. Можно задаться необходимой длиной прямолинейного участка (в интересах получения необходимой точности оценки параметров движения цели) и затем вычислить необходимые параметры манёвра. 


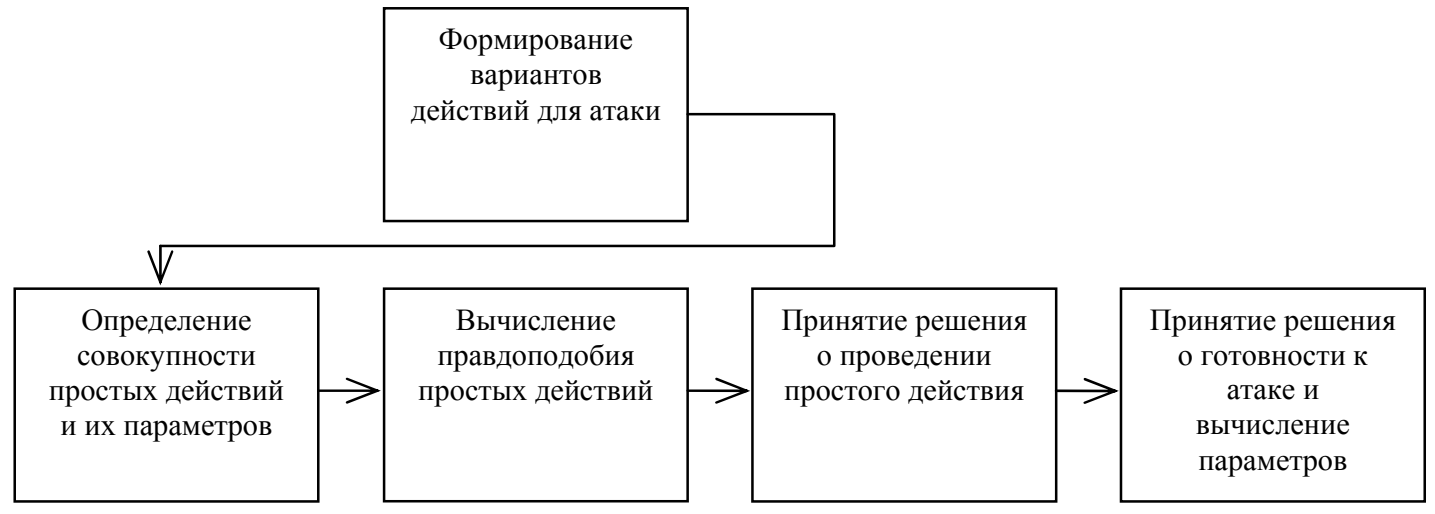

Рис. 1. Блок-схема алгоритма

Таблица 1

\begin{tabular}{|c|c|c|c|c|}
\hline $\begin{array}{c}\text { Время начала } i \text {-го } \\
\text { действия } j \text {-й цели }\end{array}$ & $\begin{array}{c}\text { Координаты } \\
\text { точки начала } i \text {-го } \\
\text { действия } j \text {-й цели }\end{array}$ & $\begin{array}{c}\text { Время конца } i \text {-го } \\
\text { действия } j \text {-й цели }\end{array}$ & $\begin{array}{c}\text { Координаты } \\
\text { точки конца } i \text {-го } \\
\text { действия } j \text {-й цели }\end{array}$ & $\begin{array}{c}\text { Угол поворота } \\
\text { в точке манёвра }\end{array}$ \\
\hline$t_{k i j}$ & $x_{n i j}$ & $t_{\kappa i j}$ & $x_{k i j} x_{i i j}$ & $\alpha_{i j}$ \\
\hline$t_{k i l}$ & $x_{n i l}$ & $t_{k i l}$ & $x_{\text {кil }}$ & $\alpha_{i l}$ \\
\hline
\end{tabular}

Возможен альтернативный вариант траектории (атака в заднюю полусферу).

5. Если истребитель проходит эту точку позже $\left(T_{\text {и0 }} \leq T_{\text {ц0}}\right)$, то он может обеспечить наведение ракеты в указанной точке путём увеличения скорости полёта. Если и в этом случае условия встречи не выполняются, то можно выполнить их в более поздних точках на траектории, используя для наведения метод погони.

6. Для маневрирующих целей параметры манёвра должны уточняться по мере получения параметров движения цели. Соответственно, уточняется граф, описывающий подготовку противника к атаке. Таким образом, в этом блоке создаётся модель движения цели (действий) для проведения атаки. Основные параметры возможных действий противника для проведения атаки используются в алгоритме оценки правдоподобия гипотез и выводятся на отображение для лётчика.

Возможный вариант отображения информации представлен в табл. 1.

В целом методы наведения перехватчиков на цели достаточно хорошо разработаны и реализованы в соответствующих АСУ, поэтому предлагаемый набор алгоритмов можно использовать как в реальных, так и в перспективных системах $[1,3,4]$.

Блок 2. В блоке проверяется соответствие получаемых траекторных измерений (параметров отдельных участков траекторных измерений, рассчитанных по данным алгоритма сопровождения), параметрам траекторий, рассчитанных в модели движения цели для проведения атаки. В связи с тем что вариантов движения в точку пуска ракет может быть много, получаемые траекторные измерения фактически уточняют тот вариант, который принят в данном конкретном случае, и на его основе вычисляются конечные параметры траектории

$$
-485-
$$


(координаты точки пуска и время пуска ракет). Степень соответствия измеренных параметров траектории и модельных определяется по значению функции правдоподобия (функционалу невязок измерений и данных модели). Информация об уточнённом варианте действий (по значению максимума функции правдоподобия) противника также выводится на средства отображения.

Основные формульные соотношения получены с использованием [5].

Пусть:

$x^{T}$ - вектор неизвестных параметров, описывающих движение цели (простое действие),

$z^{T}-$ вектор измерений (на БРЛС),

$\hat{x}^{T}$ - оценка вектора $x^{T}$.

Для Калмановского фильтра на $k$-й момент времени обновляющий сигнал, который определяется как невязки между полученными измерениями и экстраполированными координатами, определяется выражением

$$
v_{k}=z_{k}-H_{k} \hat{s}_{k / k-1},
$$

где $\hat{s}_{k}$ - оценка вектора состояния цели на $k$-й момент времени.

Матрица $H_{k}$ определяет способ объединения составляющих вектора $S_{k}$ при формировании вектора наблюдений и, следовательно, характеризует возможность определить состояние системы по результатам наблюдений.

Квадратичная форма от невязок характеризует значение логарифма функции правдоподобия (степени соответствия заложенной в фильтр модели динамической системы полученным измерениям) на $k$-м шаге

$$
J_{k}=v_{k}^{T} \Theta_{k}^{-1} v_{k},
$$

где $\Theta_{k}$ - ковариационная матрица обновляющей последовательности (рассчитывается в Калмановском фильтре).

Логарифм функции правдоподобия за $n$ шагов определяется выражением

$$
J=\sum_{k=1}^{n} j_{k}
$$

Модель движения цели в Калмановском фильтре вводится через матрицу перехода системы в новое состояние $\Phi k$

$$
s_{k}=\Phi_{k} \Phi_{k-1} \ldots \Phi_{2} \Phi_{1} s_{0} .
$$

Уравнение Калмановского фильтра, определяющее состояние системы на $k$-й момент времени,

$$
s_{k+1}=\Phi_{k} s_{k}+B_{k} u_{k}+G_{k} v_{k}
$$

включает в себя предполагаемый закон движения цели.

Блок 3. Ищем тот вариант набора параметров, характеризующих действия, который обеспечивает наибольшее значение функции правдоподобия для различных тактических приёмов, используемых противником. Функция правдоподобия для тактического приёма получается как

$$
-486-
$$


произведение функций правдоподобия для отдельных $l$-х действий (её логарифм соответственно равен сумме логарифмов функций правдоподобия):

$$
J=\sum_{l=1}^{n} j_{l}
$$

где $n$ - общее количество простых действий, входящих в тактический приём.

Тот тактический приём и те параметры приёма, которые обеспечивают максимальное значение функции правдоподобия, принимаются за наблюдаемые действия противника.

Блок 4. Фактически реализует те же принципы, что и блоки два и три, но для конечной (терминальной) ситуации - готовности к пуску ракет.

Возможные варианты графа подготовки к атаке противника представлены на рис. 2 и 3.

На графе к рис. 2: 1 - исходное состояние перехватчиков, 2 - положение перехватчиков после завершения манёвра, 3 -положение перехватчиков на момент возможного пуска ракет, $a_{12}-$ манёвр перехватчиков для выхода в точку пуска ракет, $a_{23}-$ движение по прямой в точку пуска ракет.

Граф соответствует простейшему тактическому приёму, когда группа действует без изменения боевого порядка и осуществляет пуски под произвольным углом пересечения траекторий цели и перехватчика. Меры помехового противодействия в ходе атаки не применяются.

Усложним граф, допустив, что после точки 2 из группы выделяется один перехватчик вследствие изменения модуля вектора скорости, он выходит в точку пуска ракет, а группа осуществляет информационную поддержку его действий (тактический приём «тандем»).

На графе дополнительно ко второму рисунку представлены: $a_{23}$ - манёвр перехватчика скоростью, $a_{35}$ - движение перехватчика по прямой с постоянной скоростью, $a_{24}-$ движение обеспечивающей группы по прямой с постоянной скоростью, 3 - точка завершения манёвра ударного перехватчика, 4 - положение обеспечивающей группы на момент пуска ракет, 5 - положение ударного перехватчика на момент пуска ракет.

Приведенные схемы могут быть использованы для отображения обстановки экипажу.

Место алгоритма в системе обработки и принятия решений. На вход алгоритма поступает информация об обнаружении и параметрах простых действий с выхода алгоритмов сопро-

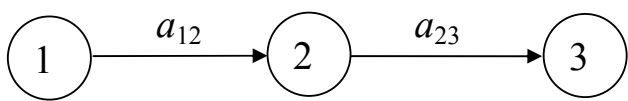

Рис. 2. Вариант 1

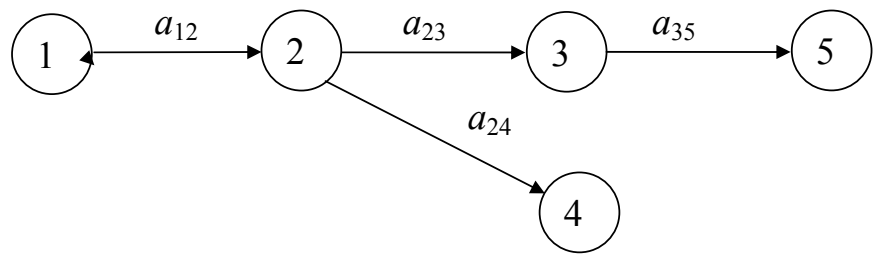

Рис. 3. Вариант 2

$$
-487-
$$


вождения, обнаружителей простых действий, измерителей параметров этих действий. Выход алгоритма используется для оценки обстановки, принятия решений на оборонительные и наступательные действия, выбора режимов работы БРЛС в интересах обнаружения пусков ракет противника.

\section{Заключение}

Таким образом, предложенный метод на основе текущей информации режима сопровождения и признаков маневрирования целей позволяет вскрыть подготовку и готовность перехватчиков противника к пуску ракет в дальнем воздушном бою, что ожидает в дальнейшем практическое подтверждение на основе результатов как имитационного (математического) моделирования исследуемой системы с использованием метода Монте-Карло, так и на основе результатов лётно-экспериментальных исследований разрабатываемых натурных алгоритмов в информационно-управляющих системах (БРЛС) модернизируемых и перспективных истребителей.

\section{Список литературы}

[1] Методы самонаведения истребителей и ракет класса «воздух-воздух» на групповую воздушную иель : монография. Ред. А. А. Кучин. Красноярск: Сиб. федер. ун-т, 2014, 168 с. [Methods homing fighters and missiles "air-air" aerial target group. Ed. by A.A.Kuchin. Krasnoyarsk, SibFU, 2014, 168 p. (in Russian)]

[2] Обнаружение и распознавание пущенной противником ракеть в бортовой радиолокаиионной станиии истребителя : монография. Ред. В. О. Черваков. Красноярск: Сиб. федер. ун-т, 2015, 160 c. [Detection and recognition of launched enemy missiles in the onboard radar of a fighter. Ed. by V.O.Chervakov. Krasnoyarsk, SibFU, 160 p. (in Russian)]

[3] Авиация ПВО России. Ред. Е.А. Федосов. Москва: Дрофа, 2004 [Air defense of Russia. Moscow, Drofa, 2004 (in Russian)]

[4] Фарина А., Студер Ф. Цифровая обработка радиолокаџионной информации. Пер. с англ. Москва: Радио и связь, 1993, 320 с. [Farina A., Studer F. A Radar Data Processing. Moscow, Radio i sviaz', 1993. 320 p (in Russian)]

[5] Замараев В.В., Лютиков И.В., Копылов В.А. Сеточный метод коллективного наведения и управляемого целераспределения группы ракет на групповую воздушную цель, оптимальный по критерию максимума вероятности её уничтожения. Журнал СФУ. Техника и технологии, 2015, 8(8), 1103-1116 [Lyutikov I.V.; Zamaraev V.V.; Shaydurov G.Y.; Kopilov, V.A.Grid Method of Collective Guidance and Controlled Target Distribution of a Group of Missiles in the Interests of Group Air Targets Effective Attack. J. Sib. Fed. Univ. Eng. technol., 2015 8(8), 1103-1116 (in Russian)] 